DOI: https://doi.org/10.24297/jap.v17i.8747

\title{
New generations of rocket engines
}

\author{
${ }^{*}$ N.J. Sobolewska ${ }^{1}$, ${ }^{*} . P$. Sobolewska ${ }^{2},{ }^{*}$ M.J. Sobolewski ${ }^{3}$, ${ }^{*}$ M.A. Sobolewski ${ }^{4},{ }^{*}$ D.S. Sobolewski ${ }^{5}$ \\ 1,2,3,4,5 Physics department, Theoretical physics, HTS High Technology Solutions, Poland \\ natalia.sobolewska@htsengines.com joanna.sobolewska@htsengines.com \\ marek.sobolewski@htsengines.com michal.sobolewski@htsengines.com \\ dariusz.sobolewski@htsengines.com
}

\begin{abstract}
The article, based on mathematical model of the space and particles revealed in "Theory of Space" (Sobolewski D. S., Theory of Space, 2016) (Sobolewski D. S., Theory of Space, 2017) and the description of the propagation of photons stated in publication entitled "Geometry of the Dark Matter and Preliminary Analysis of Alpha and Beta Photons' Properties Based on Theory of Space" (Sobolewski, Sobolewski i Sobolewski, 2017), describes transverse waves propagating in space, including gravitational, fluctuating and matter waves. In particular, it focuses on waves of matter in which orientation of the spatial channels, connecting four-dimensional boundary hypersurfaces ${ }^{\beta} \mathcal{N}^{\beta}$ and ${ }^{\alpha} \mathcal{N}^{\alpha}$, had been stabilised. For this type of the wave of matter the minimum energy value for deformation of boundary hypersurfaces of the matter waves $E_{S_{\min }}(v)$ has been appointed, which is smaller by several orders of magnitude than energy needed to change the orientation of vertical disturbances of space which connect four-dimensional boundary hypersurfaces ${ }^{\beta} \aleph^{\beta}$ and ${ }^{\alpha} \aleph^{\alpha}$.

The obtained results were used to introduce a new classification of rocket engines and to provide examples of their implementation. Furthermore, the article demonstrates first attempts to construct a rocket engine of the new type, including description of the principle of operation of the engine, which is subject of patent application entitled "HTS Photonic Rocket Engine" (Poland Patent nr P.421517, 2017), as well as presents an example of spacecraft shell implementation, which enables stabilisation of orientation of its spatial channels. The Article thus reveals new perspectives for interplanetary travel through significant increase in spacecraft velocities, while simultaneously reducing the energy required to achieve them. In addition, the article describes interaction of photons ${ }^{\beta} \gamma$ with the boundary hypersurface ${ }^{\beta}{ }^{\beta}{ }^{\beta}$, while determining the change in photon frequency depending on its curvature $\frac{v}{v_{0}}(\kappa)$.
\end{abstract}

(All co-authors provided equal contribution to the creation of this publication.)

Keywords: Rocket Engines, Gravitational Waves, Matter Waves, Fluctuating Waves, Theory Of Space

\section{Introduction}

The results presented in this publication reveal the possibilities of constructing new generation rocket engines and spacecraft, which have been unknown until now, velocity of which can even reach relativistic magnitudes, which is astounding. They are a consequence of the scientific basis formulated in "Theory of Space" (Sobolewski D. S., Theory of Space, 2016) (Sobolewski D. S., Theory of Space, 2017), as well as in the publication which describes the properties of alpha and beta photons (Sobolewski, Sobolewski i Sobolewski, 2017).

The publication proves that the structure of space and the propagation of photons, as well as other elementary particles in it, are significant in the construction of new generation rocket engines. A good example of the application of such knowledge is the structure of HTS Photon Rocket Engine (Poland Patent nr P.421517, 2017), as well as the rocket HTS engine with stabilisation, which will be discussed in this publication. 


\section{Materials and Methods}

The grounds of presented results refer to the significant theory from the theoretical physics entitled: "Theory of space" (Sobolewski D. S., Theory of Space, 2016) (Sobolewski D. S., Theory of Space, 2017), which gave us not only the mathematical model of space and particles but also introduced additional parts of energy in equation $E(v)$ :

$$
\mathrm{E}(\mathrm{v})=\mathrm{m}_{0} \mathrm{c}^{2}+\mathrm{E}_{\Delta \mathrm{J}}+\mathrm{E}_{\mathrm{S}}
$$

where $E_{\Delta \mathrm{J}}$ is the energy needed to change the orientation of vertical disturbances of space:

$$
\mathrm{E}_{\Delta \mathrm{J}}=\mathrm{m}_{0} \mathrm{c}^{2}\left(1-\cos \Theta_{\mathrm{v}}\right)
$$

while $E_{S}$ is the deformation energy of elastic boundary layers:

$$
\mathrm{E}_{\mathrm{S}}=\mathrm{m}_{0} \mathrm{c}^{2}\left(\cos ^{-1} \Theta_{\mathrm{v}}+\cos \Theta_{\mathrm{v}}-2\right)
$$

where in accordance with "Theory of Space" (Sobolewski D. S., Theory of Space, 2016) (Sobolewski D. S., Theory of Space, 2017), $\cos \Theta_{\mathrm{v}}$ is equal to:

$$
\cos \Theta_{v}=\sqrt{1-\frac{v^{2}}{c^{2}}}
$$

Thus, in this work method of analysis and logical structure have been applied based on notions, models and equations introduced by the "Theory of Space", along with the article entitled: "Geometry of the Dark Matter and Preliminary Analysis of Alpha and Beta Photons' Properties Based on Theory of Space" that introduces initial results of analysis of the properties of space channels connecting the same border hypersurfaces of fourdimensional differential manifold immersed in a four-dimensional Euclidean space. (Sobolewski, Sobolewski i Sobolewski, 2017)

\section{Results and Discussion}

\section{Classification of waves}

Classification of the existing transverse waves has been introduced based on the mathematical model of space and particles introduced in the "Theory of Space" (Sobolewski D. S., Theory of Space, 2016) (Sobolewski D. S., Theory of Space, 2017) that revealed elastic properties of boundary hypersurfaces of space, which enable classification of the transverse waves propagating in space into:

- Gravitational waves - which change distances between boundary hypersurfaces of the space without spatial channels,

- Matter waves - which lift the spatial channels,

- Fluctuating waves - which do not change distances between boundary hypersurfaces without spatial channels.

Please notice that the understanding of all types of waves propagating in space is solid grounds for the development of the unified quantum mechanics theories. 


\section{Minimum of the deformation energy of matter waves}

The minimum energy of deformed boundary hypersurfaces $E_{S_{\min }}(v)$ has been calculated for the matter waves which lift the spatial channels having mass $\mathrm{m}_{0}$ with the velocity $v$ and with stabilised orientation of the spatial channels, connecting four-dimensional boundary hypersurfaces ${ }^{\beta} \aleph^{\beta}$ and ${ }^{\alpha} \aleph^{\alpha}$ :

$$
E_{S_{\text {min }}}(v) \equiv \mathrm{m}_{0} \mathrm{c}^{2}\left(\cos \Theta_{\mathrm{v}}-1\right)^{2}=\mathrm{m}_{0} \mathrm{c}^{2}\left(1-\sqrt{1-\frac{v^{2}}{c^{2}}}\right)^{2}
$$

The energy $E_{S_{\min }}(v)$ can be treated also as minimum of energy needed for space rocket with mass $\mathrm{m}_{0}$ to achieve the speed $v$ in case if the spatial channels of the space rocket will not change the angle of inclination.

In this way it was theoretically proven that energy needed for increasing speed of the body with the mass $\mathrm{m}_{0}$ is not limitless but finite even for relativistic speeds what was shown on the figure Fig. 1.

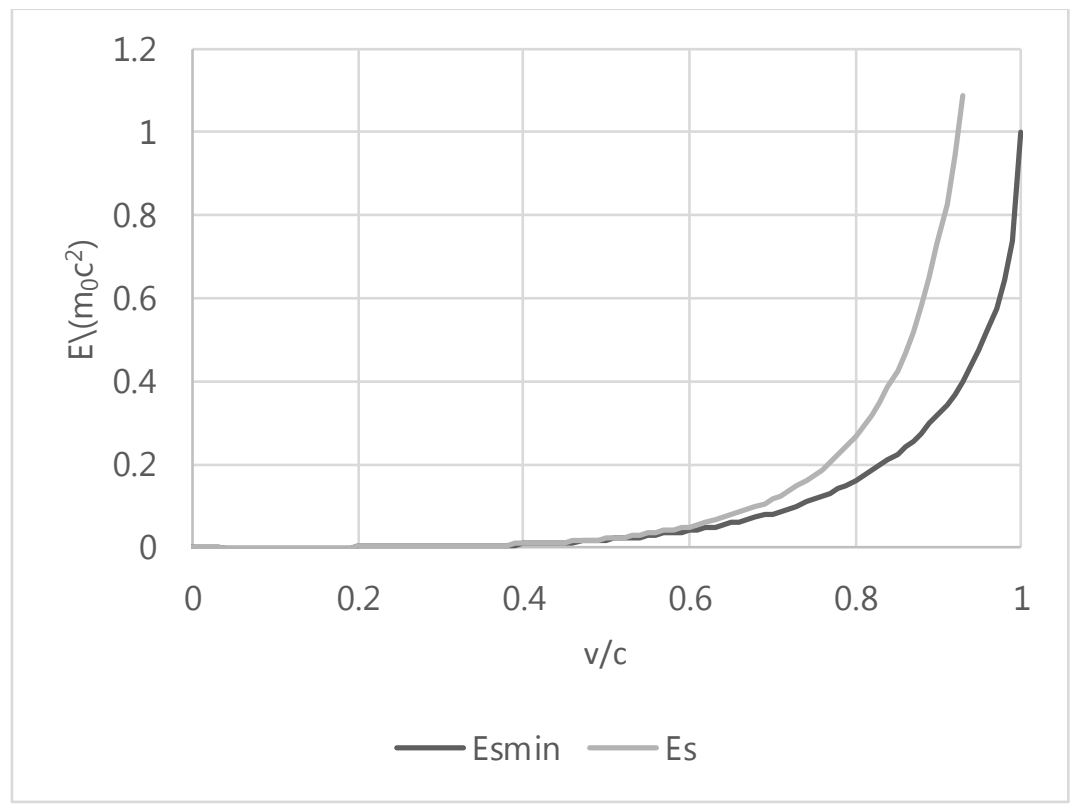

Fig. 1 Graph presenting the dependence of $\frac{E_{S_{\min }\left(\frac{v}{c}\right)}}{m_{0} c^{2}}$ and $\frac{E_{S}\left(\frac{v}{c}\right)}{m_{0} c^{2}}$

\section{Classification of the rocket engines}

Taking the nature of matter waves propagation into account, a division methodology for rocket engines has been introduced:

- Jet engines,

- HTS engines,

- $\quad$ HTS engines with stabilisation.

Jet rocket engines change the orientation of space channels, from which the spacecraft is constructed, and as a result of that, the deformation of boundary hypersurfaces ${ }^{\beta} \aleph^{\beta}$ and ${ }^{\alpha} \aleph^{\alpha}$ changes. On the other hand, HTS Rocket Engines change the deformation of boundary hypersurfaces ${ }^{\beta} \mathrm{N}^{\beta}$ and ${ }^{\alpha} \mathrm{N}^{\alpha}$ of the spacecraft, which results in a change in the orientation of its spatial channels. 
HTS Rocket Engines with stabilisation are HTS engines, which by changing the deformation of boundary hypersurfaces ${ }^{\beta} \aleph^{\beta}$ and ${ }^{\alpha} \aleph^{\alpha}$, do not change the orientation of spatial channels from which the spacecraft is constructed. It should be underlined that shield of the rocked engine and the spacecraft has been accounted as a part of the HTS engine with stabilisation.

\section{Equation for the photon frequency change}

The following formula for determining the change in photon frequency in space curvature was introduced ${ }^{1}$ :

$$
\frac{v}{v_{0}} \equiv 1-\frac{2}{\pi} \int_{s_{0}}^{s_{1}} \kappa(s) d s
$$

where the curvature $\kappa$ :

$$
\kappa \equiv \lim _{\Delta S \rightarrow 0} \frac{\Delta \alpha}{\Delta S}
$$

which for the parametrically described function is expressed by the following formula:

$$
\kappa(t)=\frac{y^{\prime \prime}(t) x^{\prime}(t)-x^{\prime \prime}(t) y^{\prime}(t)}{\left(x^{\prime}(t)^{2}+y^{\prime}(t)^{2}\right)^{\frac{3}{2}}}
$$

Formula introduced in equation (6) can be used for calculation of energy losses of photons propagating in curved space. Furthermore, the formula (6) used for the photons reaching Earth from far away galaxies clearly shows the so called "red-shifts" in spectral lines and underlines the limitation of the area of the Universe, which is visible to us, to half-sphere with radius $W_{\Re}=4040.33 M p c$, (Sobolewski, Sobolewski i Sobolewski, 2017) (Sobolewski D. S., Theory of Space, 2016) (Sobolewski D. S., Theory of Space, 2017) what was shown on the figure Fig. 2.

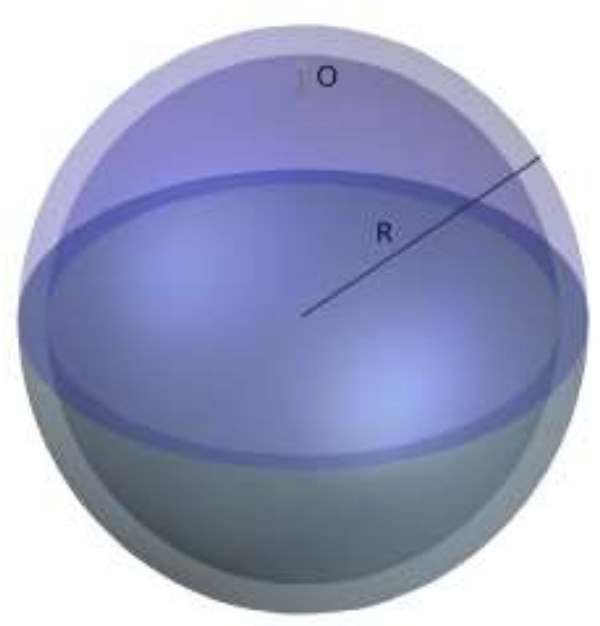

Fig. 2 Visible area of the Universe from the $O$ point.

\footnotetext{
${ }^{1}$ In subsequent publications, we will introduce more general formulas and the geometric concept of four-dimensional curvature of the boundary hypersurface.
} 


\section{Theoretical possibilities of building HTS engines with stabilisation}

The theoretical possibilities of building HTS engines with stabilisation of the orientation of spatial channels from which the spacecraft is constructed has been considered to reduce energy $E_{\Delta \mathrm{J}}$, which is needed to change orientation of the spatial channels connected boundary hypersurfaces ${ }^{\beta} \mathrm{N}^{\beta}$ and ${ }^{\alpha} \aleph^{\alpha}$, to nearly zero.

Such a theoretical solution has been achieved by adding additional deformation $\psi$ of boundary hypersurfaces ${ }^{\beta} \aleph^{\beta}$ and ${ }^{\alpha} \aleph^{\alpha}$ around the spacecraft, which is shown on the figure Fig. 3.

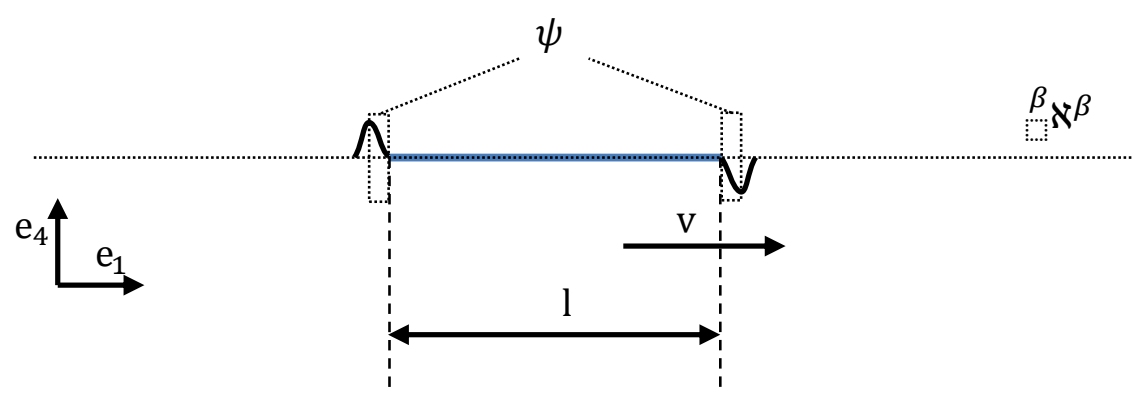

Fig. 3 Spacecraft with mass $m_{0}$ which moves at velocity $v$, covered with additional deformation $\psi$ of boundary hypersurfaces ${ }^{\beta} \aleph^{\beta}$ and ${ }^{\alpha} \aleph^{\alpha}$ around rocket - overview figure.

Efforts related with the attempt to minimize energy $E_{\Delta \mathrm{J}}$ are visible on graph in the figure Fig. 4 which presents the dependency $\frac{E_{\Delta J}}{E_{S_{\min }}}(v)$ shown in the equation (9).

$$
\frac{E_{\Delta \mathrm{J}}}{E_{S_{\min }}}=\frac{\mathrm{m}_{0} \mathrm{c}^{2}\left(1-\cos \Theta_{\mathrm{v}}\right)}{\mathrm{m}_{0} \mathrm{c}^{2}\left(\cos \Theta_{\mathrm{v}}-1\right)^{2}}=\frac{1}{1-\cos \Theta_{\mathrm{v}}}=\frac{1}{1-\sqrt{1-\frac{v^{2}}{c^{2}}}}
$$

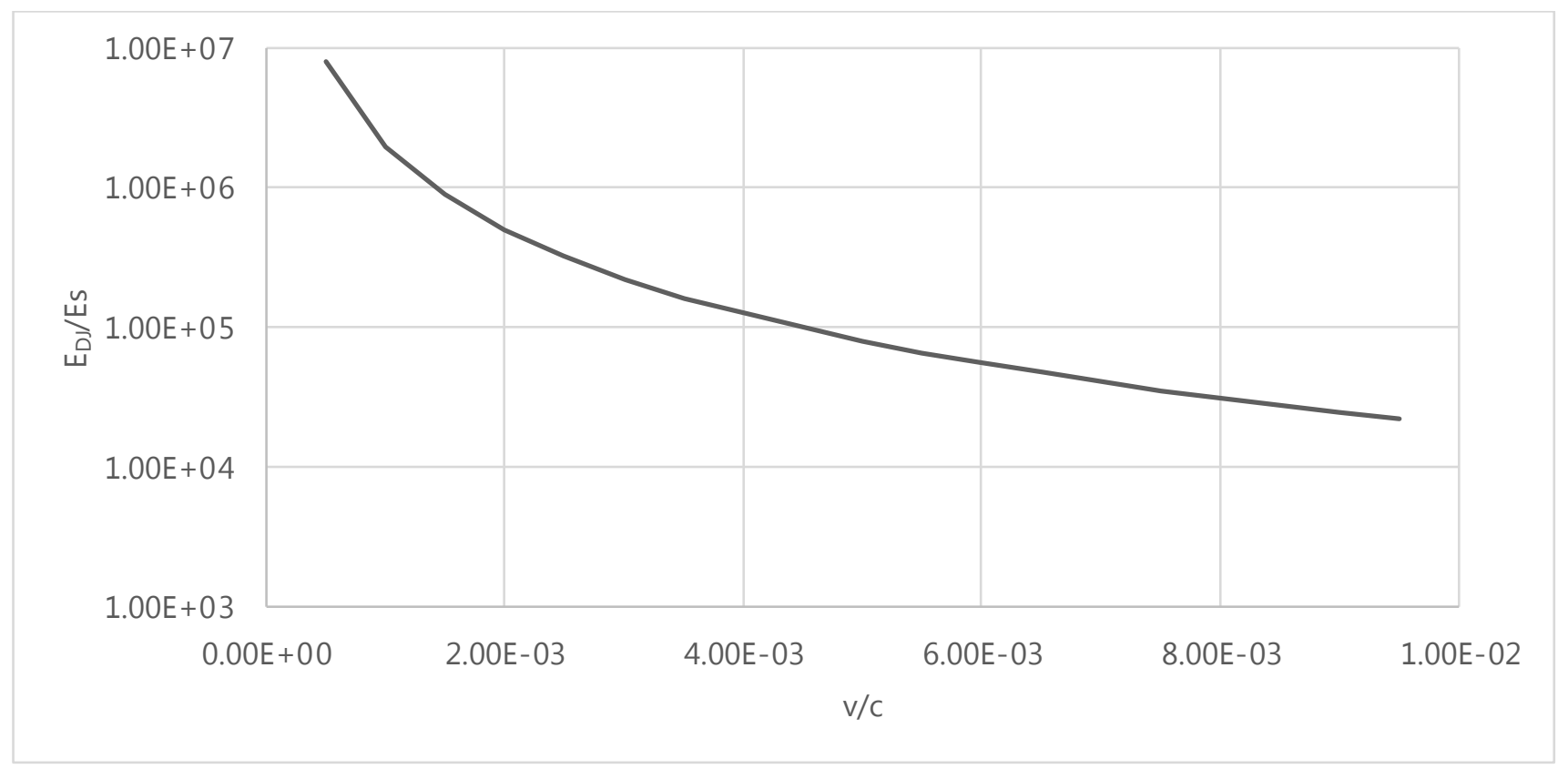

Fig. 4 Graph of dependence $\frac{E_{\Delta J}}{E S_{\min }}\left(\frac{v}{c}\right)$ 


\section{An example of implementation of HTS engine with stabilisation}

An example of implementation of HTS engine with stabilization has been revealed. Additional deformation $\psi$ of boundary hypersurfaces ${ }^{\beta} \aleph^{\beta}$ and ${ }^{\alpha} \aleph^{\alpha}$ shown on the figure Fig. $\mathbf{3}$ around rocket is achieved by the application of electrically isolated graphene layers as a spacecraft shell, through which the current would flow in manner marked on Fig. 5 below.

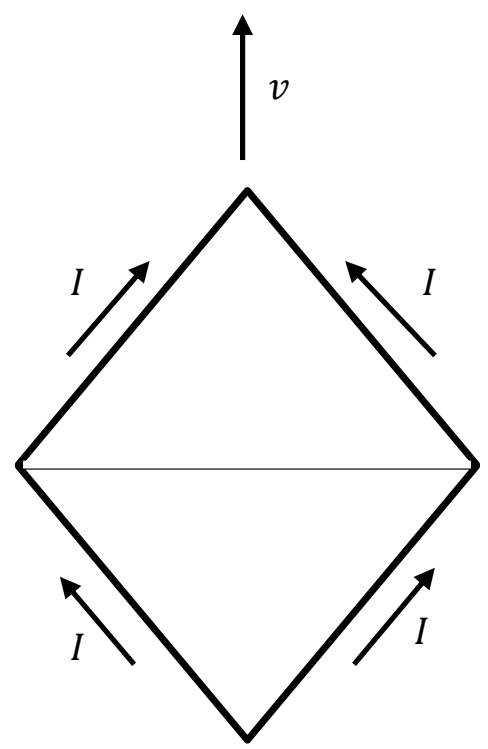

Fig. 5 Overview figure of a spacecraft covered with the layers of graphene, through which the electric current $I$ flows.

By applying additional deformation $\psi$ of boundary hypersurfaces ${ }^{\beta} \boldsymbol{\aleph}^{\beta}$ and ${ }^{\alpha} \boldsymbol{\aleph}^{\alpha}$ shown on the figure Fig. 3 we avoid propagation of the elastic deformation $\xi$ of boundary hypersurfaces ${ }^{\beta} \aleph^{\beta}$ and ${ }^{\alpha} \aleph^{\alpha}$, created by the HTS engine, along the whole spacecraft what is shown on the figure Fig. 6.

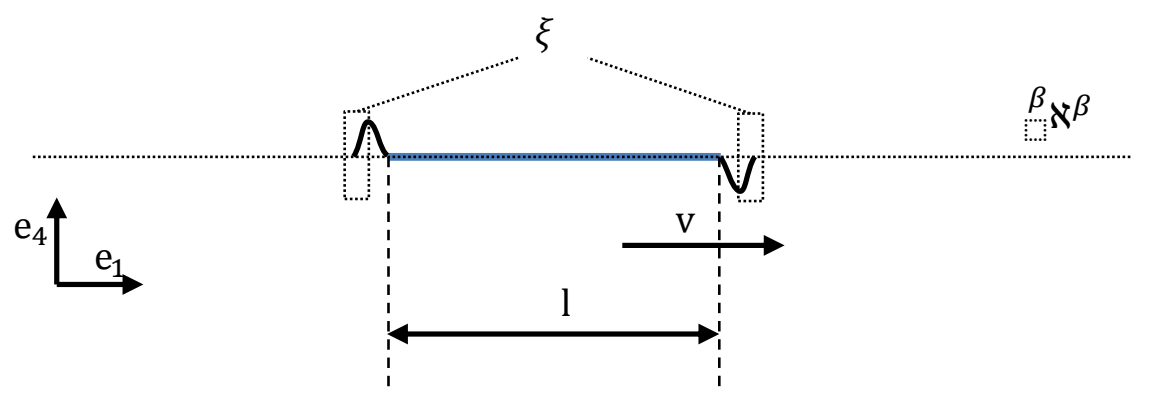

Fig. 6 Spacecraft with mass $m_{0}$ that moves at velocity $v$, covered with elastic deformation $\xi$ of the boundary hypersurfaces ${ }^{\beta} \aleph^{\beta}$ and ${ }^{\alpha} \aleph^{\alpha}$ created by the HTS engine - overview figure.

Space deformations (Main Text): It results directly from the theory entitled "Theory of Space" (Sobolewski D. S., Theory of Space, 2016) (Sobolewski D. S., Theory of Space, 2017), that the mathematical model of physical space is a smooth four-dimensional differential manifold ${ }^{R e} M$, which is embedded in the four-dimensional Euclidean space $\mathrm{E}^{4}$. 
Differential manifold ${ }^{R e} M$ is limited by three-dimensional boundary hypersurfaces in shape of spheres, whose radius is equal to ${ }^{w} \mathfrak{R}$, while the distance between them on the Earth's surface amounts to $\tau_{E}$ (Sobolewski D. S., Theory of Space, 2016) (Sobolewski D. S., Theory of Space, 2017):

$$
\begin{gathered}
\mathrm{w}_{\Re} \cong \frac{\mathrm{c}}{\mathrm{H}_{0}} \cong 4040,33 \mathrm{Mpc} \\
\tau_{E} \geq 0,894 \mu \mathrm{m}
\end{gathered}
$$

Motion of the elementary particles, which are essentially vortical disturbances of space, is possible thanks to the asymmetric deformation of four-dimensional boundary layers of the differential manifold ${ }^{R e} M$ (Sobolewski D. S., Theory of Space, 2016) (Sobolewski D. S., Theory of Space, 2017).

On the other hand, the vortical disturbances themselves create tubes (which are also called spatial channels) that connect the same ( $\alpha$ and $\beta$ photons) and different boundary hypersurfaces (quarks and $p$-quarks) of the differential manifold ${ }^{R e} M$ (Sobolewski D. S., Theory of Space, 2016) (Sobolewski D. S., Theory of Space, 2017), which is presented in the Fig. 1.

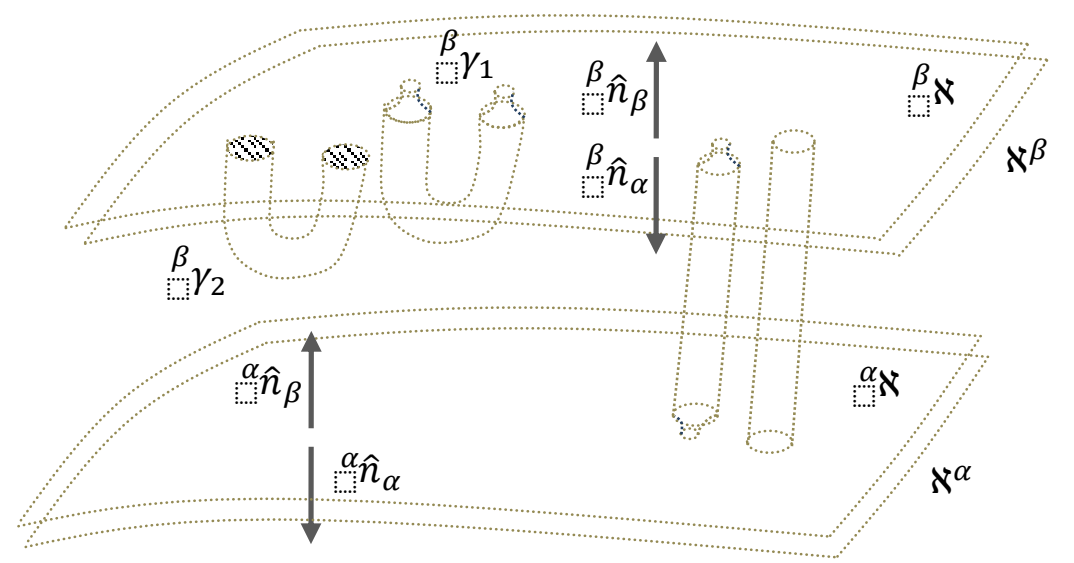

Fig. $7 \boldsymbol{\beta}$ photons, quarks and $\mathbf{p}$-quarks in the differential manifold ${ }^{R e} M$

The moving spatial channels oscillate around the equilibrium position inclined at an angle $\Theta$, depending on its velocity, in accordance with the following equation (Sobolewski D. S., Theory of Space, 2016) (Sobolewski D. S., Theory of Space, 2017):

$$
\theta=\arcsin \left(\frac{v}{c}\right)
$$

that also perform nutations, which contemporary quantum mechanics equate to the matter waves.

If the moving object is a macroscopic one, which is built of a very large number of spatial channels, we will assume, in order to simplify the considerations, that the macroscopic objects are rigid bodies.

In case of such an assumption, a macroscopic object with mass $m_{0}$, which moves in the direction of axis $e_{1}$ at velocity $v$, deforms four-dimensional elastic boundary layers ${ }^{\beta} \aleph^{\beta}$ and ${ }^{\alpha} \aleph^{\alpha}$ that have three-dimensional boundary hypersurfaces ${ }^{\beta} \mathrm{N}$ and $\aleph^{\beta}$, and ${ }^{\alpha} \mathrm{N}$ and $\mathrm{K}^{\alpha}$, respectively, as presented in the overview Fig. 8 . 


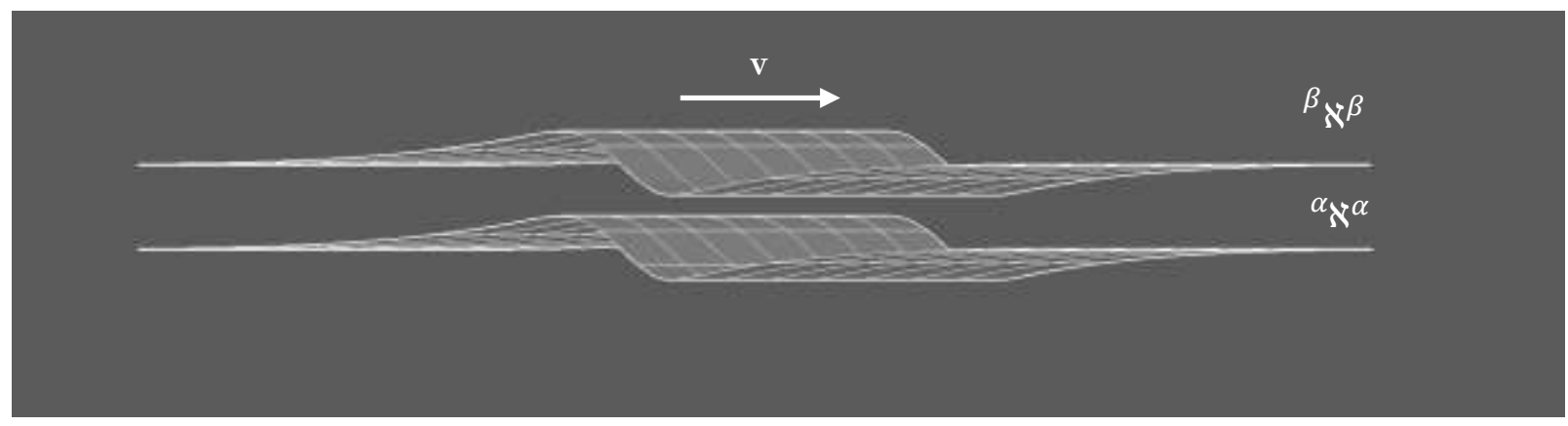

Fig. 8 Macroscopic object moving in the direction of axis $e_{1}$ at velocity $v$-overview figure.

It results directly from the theory entitled "Theory of Space" that the dependence of energy of such object on the velocity E(v) is given with the following formula (Sobolewski D. S., Theory of Space, 2016) (Sobolewski D. S., Theory of Space, 2017):

$$
\mathrm{E}(\mathrm{v})=\mathrm{m}_{0} \mathrm{c}^{2}+\mathrm{E}_{\Delta \mathrm{J}}+\mathrm{E}_{\mathrm{S}}
$$

where $E_{\Delta \mathrm{J}}$ is the energy needed to change the orientation of vortical disturbances of space:

$$
\mathrm{E}_{\Delta \mathrm{J}}=\mathrm{m}_{0} \mathrm{c}^{2}\left(1-\cos \Theta_{\mathrm{v}}\right)
$$

while $E_{S}$ is the deformation energy of elastic boundary layers:

$$
\mathrm{E}_{\mathrm{S}}=\mathrm{m}_{0} \mathrm{c}^{2}\left(\cos ^{-1} \Theta_{\mathrm{v}}+\cos \Theta_{\mathrm{v}}-2\right)
$$

where, in accordance with the theory entitled "Theory of Space", $\cos \Theta_{\mathrm{v}}$ is equal to: (Sobolewski D. S., Theory of Space, 2016) (Sobolewski D. S., Theory of Space, 2017)

$$
\cos \Theta_{\mathrm{v}}=\sqrt{1-\frac{v^{2}}{c^{2}}}
$$

It is necessary to note that the orientation change of four-dimensional angular momentum is identified with the object's classical momentum $\mathrm{p}_{\Delta \mathrm{J}}$ (Sobolewski D. S., Theory of Space, 2016) (Sobolewski D. S., Theory of Space, 2017):

$$
\mathrm{p}_{\Delta \mathrm{J}}=\mathrm{m}_{0} \mathrm{v}
$$

while energy of the deformed boundary layers $E_{S}$ is identified with momentum $p_{S}$ :

$$
\mathrm{p}_{\mathrm{s}}=\mathrm{m}_{0} \mathrm{v}\left(\cos ^{-1} \Theta_{\mathrm{v}}-1\right)
$$

As an example, let us consider a spacecraft with mass $\boldsymbol{m}_{\mathbf{0}}=\mathbf{1 0 0 0} \mathbf{k g}$ and length $\boldsymbol{l}=\mathbf{2 0} \mathbf{m}$, which moves at velocity $v=30 \frac{\mathrm{km}}{\mathrm{s}}$ and let us determine from the equation (11) its angle of inclination $\boldsymbol{\Theta}_{\mathbf{v}}$ in relation to the boundary hypersurfaces:

This is presented in a simplified manner on Fig. 9.

$$
\theta=\arcsin \left(\frac{v}{c}\right)=\arcsin \left(\frac{30 * 10^{3} \frac{\mathrm{m}}{\mathrm{s}}}{299792458 \frac{\mathrm{m}}{\mathrm{s}}}\right) \approx \arcsin \left(10^{-4}\right) \approx 10^{-4} \mathrm{rad}
$$




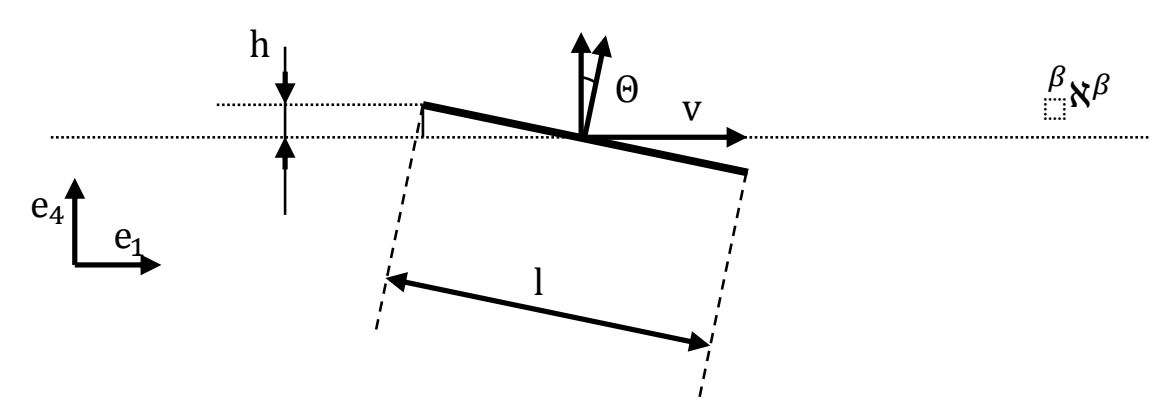

Fig. 9 Spacecraft ship with mass $m_{0}$ that moves at velocity $v$-overview figure.

Deformation height $h$ of the boundary hypersurface ${ }^{\beta} \aleph^{\beta}$ for a spacecraft with length $l=20 m$, with the assumption that it does not deform during flight ${ }^{2}$, amounts to:

$$
h=\frac{1}{2} * \sin \theta \cong \frac{20 \mathrm{~m}}{2} * 10^{-4}=10^{-3} \mathrm{~m}=1 \mathrm{~mm}
$$

The formula (13) is used to determine the energy $E_{\Delta \mathrm{J}}$ needed to change the orientation of spatial channels by angle $\theta$ during acceleration of the spacecraft:

$$
\begin{aligned}
\mathrm{E}_{\Delta \mathrm{J}}=\mathrm{m}_{0} \mathrm{c}^{2}(1- & \left.\cos \Theta_{\mathrm{v}}\right)=\mathrm{m}_{0} \mathrm{c}^{2}\left(1-\sqrt{1-\frac{v^{2}}{c^{2}}}\right) \\
& \approx 10^{3} \mathrm{~kg} *\left(3 * 10^{8} \frac{\mathrm{m}}{\mathrm{s}}\right)^{2}\left(1-\sqrt{1-\left(\frac{30 * 10^{3} \frac{\mathrm{m}}{\mathrm{s}}}{3 * 10^{8} \frac{\mathrm{m}}{\mathrm{s}}}\right)^{2}}\right) \\
& \approx 4.5 * 10^{11} \mathrm{~J}
\end{aligned}
$$

Ultimately, the energy $E_{\Delta J}$ required to change the orientation of spacecraft's space channels by the angle $\theta$ is equal to $450 \mathrm{GJ}$.

By using the formula (14), it is possible to determine the energy of elasticity $E_{S}$ of boundary hypersurfaces deformed by this spacecraft:

$$
\mathrm{E}_{\mathrm{S}}=\mathrm{m}_{0} \mathrm{c}^{2}\left(\cos ^{-1} \Theta_{\mathrm{v}}+\cos \Theta_{\mathrm{v}}-2\right)=9 * 10^{19} * 2.5 * 10^{-17} \mathrm{~J}=2.25 * 10^{3} \mathrm{~J}
$$

Therefore, the potential energy of elasticity of the boundary hypersurfaces ${ }^{\alpha} \boldsymbol{\aleph}^{\alpha}$ and ${ }^{\beta} \boldsymbol{\aleph}^{\beta}$ deformed by a spacecraft with the mass of one ton moving at velocity of $30 \frac{\mathrm{km}}{\mathrm{s}}$ amounts to only $2.25 \mathrm{~kJ}$.

Let us calculate the quotient $\frac{E_{\Delta J}}{E_{S}}$ :

\footnotetext{
2 The spacecraft is constructed from a large number of spatial channels that connect various boundary hypersurfaces, which at the length $\boldsymbol{l}$ can significantly deform the boundary hypersurfaces and for that reason, the height $\mathrm{h}$ can be significantly smaller.
} 


$$
\frac{\mathrm{E}_{\Delta \mathrm{J}}}{E_{S}} \approx \frac{4.5 * 10^{11} \mathrm{~J}}{2.25 * 10^{3} \mathrm{~J}}=2 * 10^{8}
$$

This means that the energy $\mathrm{E}_{\Delta \mathrm{J}}$, required to change the orientation of the spacecraft's space channels with mass $\mathrm{m}=10^{3} \mathrm{~kg}$ by an angle $\theta=10^{-4} \mathrm{rad}$, is as much as eight orders of magnitude greater than the energy of elasticity of the deformed boundary hypersurfaces, which are in fact responsible for the spacecraft's motion, which will be discussed in the next chapter.

\section{Matter waves, gravitational waves and fluctuating waves}

The structure of space and elementary particles revealed by the theory entitled "Theory of Space" (Sobolewski D. S., Theory of Space, 2016) (Sobolewski D. S., Theory of Space, 2017) forces the revision of the basic concepts of physics, including the concept of motion. Motion is still a relative concept, but in relation to the space, which is not enigmatic ether but has a layered structure, including boundary layers with elastic properties, which are limited by three-dimensional boundary hypersurfaces.

Thanks to the elastic properties of four-dimensional boundary hypersurfaces ${ }^{\alpha} \aleph^{\alpha}$ and ${ }^{\beta}{ }^{\beta}$ of the space, it is possible for the transverse waves to propagate in it.

At the same time, the transverse waves that change the distances between the boundary hypersurfaces ${ }^{\beta} \aleph^{\beta}$ and ${ }^{\alpha} \aleph^{\alpha}$ will be equated with the gravitational waves, in accordance with the TP theory, and it should be noted that these waves were predicted by A. Einstein.

In general, we can divide the transverse waves propagating in space into:

- Gravitational waves - which change distances between boundary hypersurfaces of the space without spatial channels,

- Matter waves - which lift the spatial channels,

- Fluctuating waves - which do not change distances between boundary hypersurfaces without spatial channels.

The introduced space fluctuating waves constitute a consequence of the structure of physical space, disclosed in the theory entitled "Theory of Space" (Sobolewski D. S., Theory of Space, 2016) (Sobolewski D. S., Theory of Space, 2017). They can be generated similarly to the gravitational waves by the binary systems of astronomical objects, including black holes or supernova explosions. However, unlike in the case of gravitational waves, the principle of wave superposition should apply to this type of waves ${ }^{3}$.

Similarly to the gravitational waves, the fluctuating waves should propagate at the velocity of light and interact with matter (spatial channels). The difference between fluctuating waves and gravitational waves should affect their range of propagation. Moreover, from the theoretical viewpoint, there is a possibility of transformation of the fluctuating waves into gravitational waves and vice versa, along with the distance travelled by the wavefront.

\footnotetext{
${ }^{3}$ In the case of gravitational waves, we are dealing with a variable distance between the boundary hypersurfaces ${ }^{\beta} \aleph^{\beta}$ and ${ }^{\alpha} \aleph^{\alpha}$, thus the principle of wave superposition is not applicable to them.
} 


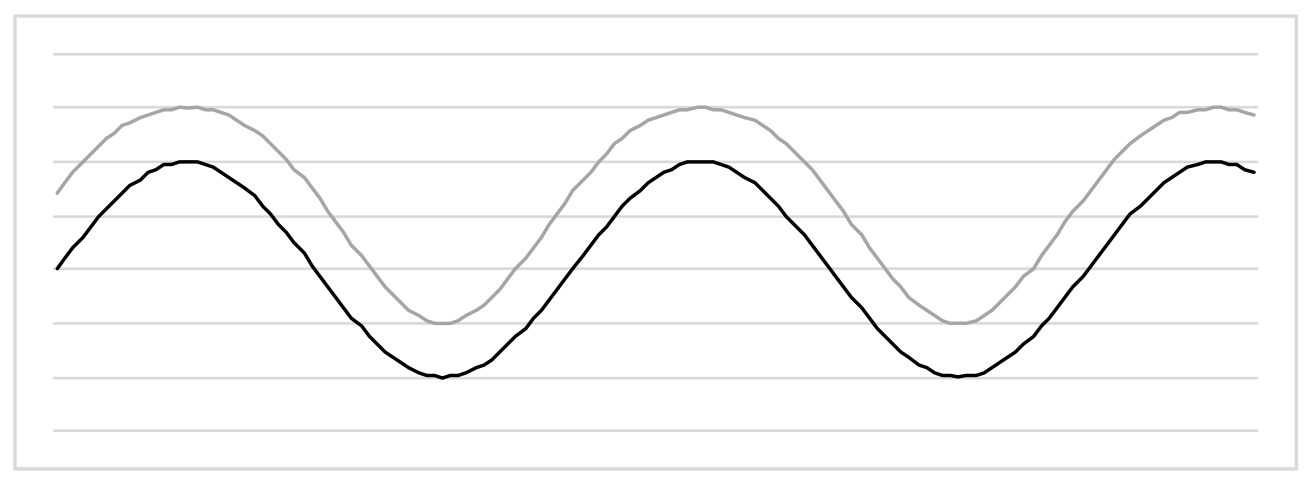

Fig. 10 Fluctuating waves $^{4}$ - overview figure.

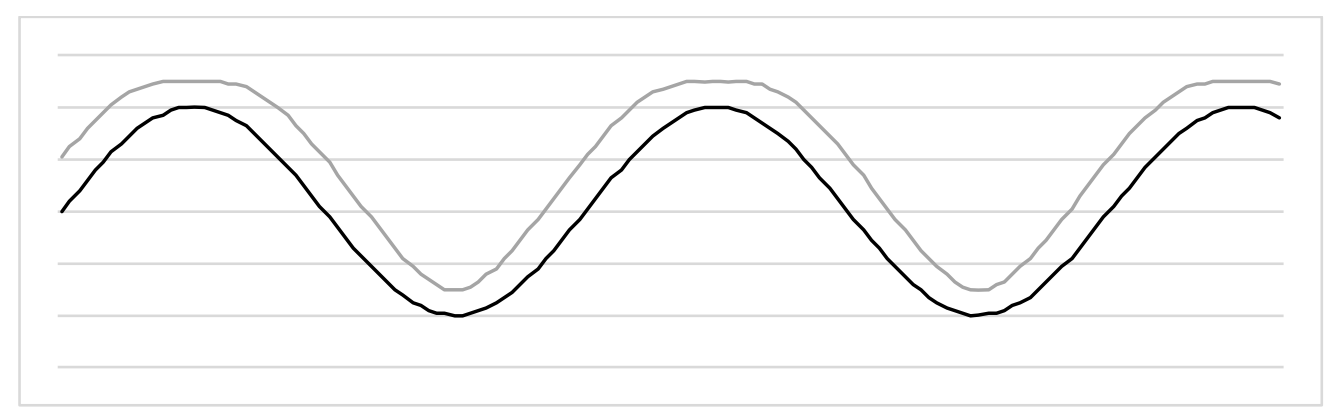

Fig. 4 Gravitational waves (Sobolewski D. S., Theory of Space, 2016) (Sobolewski D. S., Theory of Space, 2017) - overview figure.

It should be emphasised that due to the variable distance between the boundary hypersurfaces ${ }^{\beta} \aleph^{\beta}$ and ${ }^{\alpha} \aleph^{\alpha}$, gravitational waves will not satisfy the principle of superposition.

Moreover, it should be noted that the range of gravitational waves is significantly greater in the case of applying a variable distance between the boundary hypersurfaces ${ }^{\beta} \mathrm{N}^{\beta}$ and ${ }^{\alpha} \aleph^{\alpha}$ on a significantly greater transverse deformation of the physical space. This fact should be used to explain the possibility of detecting gravitational waves, generated by rotating binary systems of astronomical objects.

In the subsequent part of this article, we will discuss only the matter waves, which are presented in the Fig. 8 , Fig. 9.

The waves of this type accompany a motion of the vortical space disturbances that the matter is made of, therefore the interactions between elastic boundary hypersurfaces of the space and the moving space vortical disturbances lead to the oscillation of spatial channels and their nutations, which are equated to the matter waves, in accordance with De Broglie equation:

${ }^{4}$ In order to make a graph, the formulas were used in regard to the function: ${ }^{\beta} \chi\left(X^{1}\right)=\left(X^{1}\left(x^{1}\right), X^{4}\left(x^{1}\right)\right)$ for ${ }^{\alpha} \chi\left(x^{1}\right)=\sin \left(x^{1}\right)$, where $X^{1}=x^{1}-\tau \frac{d^{\alpha} \chi\left(x^{1}\right)}{d x^{1}} \frac{1}{\sqrt{1+\left(\frac{d^{\alpha} \chi\left(x^{1}\right)}{d x^{1}}\right)^{2}}}$ and $X^{4}={ }^{\alpha} \chi\left(x^{1}\right)+\tau \frac{1}{\sqrt{1+\left(\frac{d^{\alpha} \chi\left(x^{1}\right)}{d x^{1}}\right)^{2}}}$ for a constant distance between the boundary hypersurfaces $\tau=0.5$ (Sobolewski D. S., Theory of Space, 2016) (Sobolewski, Sobolewski i Sobolewski, 2017). 


$$
\lambda=\frac{h}{p}=\frac{h}{m v}
$$

where:

$h$ - Planck's constant,

$p$ - momentum of the elementary particle,

$m$ - mass of the elementary particle,

$v$ - velocity of the elementary particle.

The equations of motion for a single spatial channel are presented in the theory entitled "Theory of Space", originating from the Lagrangian function (Sobolewski D. S., Theory of Space, 2016) (Sobolewski D. S., Theory of Space, 2017):

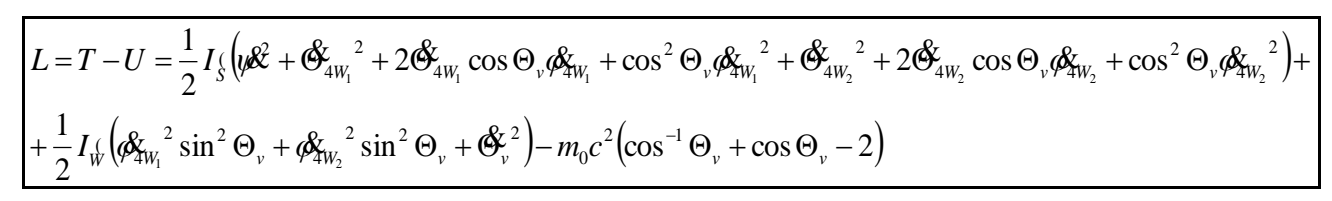

where the potential energy $\mathrm{U}$ is the deformation energy of boundary hypersurfaces:

$$
\mathrm{U}=E_{S}=\mathrm{m}_{0} \mathrm{c}^{2}\left(\cos ^{-1} \Theta_{\mathrm{v}}+\cos \Theta_{\mathrm{v}}-2\right)
$$

In the case when the moving object is a macroscopic rigid body, the deformations of the boundary hypersurfaces ${ }^{\alpha} \aleph^{\alpha}$ and ${ }^{\beta} x^{\beta}$ add up for the individual vortical space disturbances ${ }^{5}$, an overview of which is presented on Fig. 8.

Direct cause of the motion of matter is the deformation of boundary hypersurfaces ${ }^{\alpha} \aleph^{\alpha}$ and ${ }^{\beta} \aleph^{\beta}$, i.e. a transverse wave, whose vibration direction that is perpendicular in relation to the direction of wave propagation lifts the vortical space disturbances, which are trapped in the potential well.

It is necessary to note that the transverse wave cannot force an area with vortical space disturbances, because it would have to change their orientation and relative position along the axis $e^{4}$, which would require significantly greater energy. For the same reason, the vortical space disturbances are trapped between the deformed boundary hypersurfaces.

The transverse deformation of the boundary hypersurfaces ${ }^{\alpha} \aleph^{\alpha}$ and ${ }^{\beta} \boldsymbol{\aleph}^{\beta}$ of the matter waves cannot propagate at the velocity of light, because it is blocked by vortical space disturbances, which are trapped in the potential well. Thus, a question arises: what is the minimum energy value of deformed boundary hypersurfaces, so that the vortical space disturbances would move at a given velocity $v$-just like a "surfer" on the wave.

For this purpose, we will expand the function $E_{S}\left(\cos \Theta_{v}\right)$ from the formula (14) into a Taylor series for $\cos \Theta_{v}=1$ :

${ }^{5}$ Theory entitled "Theory of Space" identifies the vortical space disturbances with matter (Sobolewski D. S., Theory of Space, 2016) (Sobolewski D. S., Theory of Space, 2017).

${ }^{6}$ Spatial channels cannot pass through the deformed boundary hypersurfaces, because they would have to change their orientation, i.e. $\mathrm{E}_{\Delta \mathrm{J}}$. 


$$
\begin{aligned}
& E_{S}=\mathrm{m}_{0} \mathrm{c}^{2}\left(\cos ^{-1} \Theta_{\mathrm{v}}+\cos \Theta_{\mathrm{v}}-2\right) \approx \\
& \mathrm{m}_{0} \mathrm{c}^{2}\left(\cos \Theta_{\mathrm{v}}-1\right)^{2}-\mathrm{O}\left(\left(\cos \Theta_{\mathrm{v}}-1\right)^{3}\right)
\end{aligned}
$$

Ultimately, by substituting $\cos \Theta_{v}$ with right side of the equation (15) and limiting to only one element of the series, we can determine the minimum energy of deformed boundary hypersurfaces for a given spacecraft velocity $\mathrm{v}$ :

$$
E_{S_{\text {min }}}(v) \equiv \mathrm{m}_{0} \mathrm{c}^{2}\left(\cos \Theta_{\mathrm{v}}-1\right)^{2}=\mathrm{m}_{0} \mathrm{c}^{2}\left(1-\sqrt{1-\frac{v^{2}}{c^{2}}}\right)^{2}
$$

Then another question arises: what is the physical sense of the limitation applied to small values of the angle $\Theta_{\mathrm{v}}$ ?

The answer results directly from the previous chapter, because we do not want to change the orientation of spatial channels, in order to save significant amounts of energy, which are necessary to accelerate the spacecraft to the velocity $v$. Therefore, during acceleration of the spacecraft, it will be necessary to stabilise orientation of the spatial channels, in order to ensure that $E_{\Delta J}$ is close to zero.

It results directly from the formula (26) that in order to accelerate a spacecraft to the velocity of light $v=c$, in which the vortical space disturbances do not change orientation ${ }^{7}$, it is necessary to obtain the deformation energy of boundary hypersurfaces equal to $\mathrm{m}_{0} \mathrm{c}^{2}$.

It is worth to illustrate the obtained result by the graph of energy per mass unit $E_{S_{\min }}^{\prime}$, which determines the minimum theoretical energy values required to accelerate a spacecraft to the velocity $v$, while assuming the velocity of light $c$ as a unit of velocity.

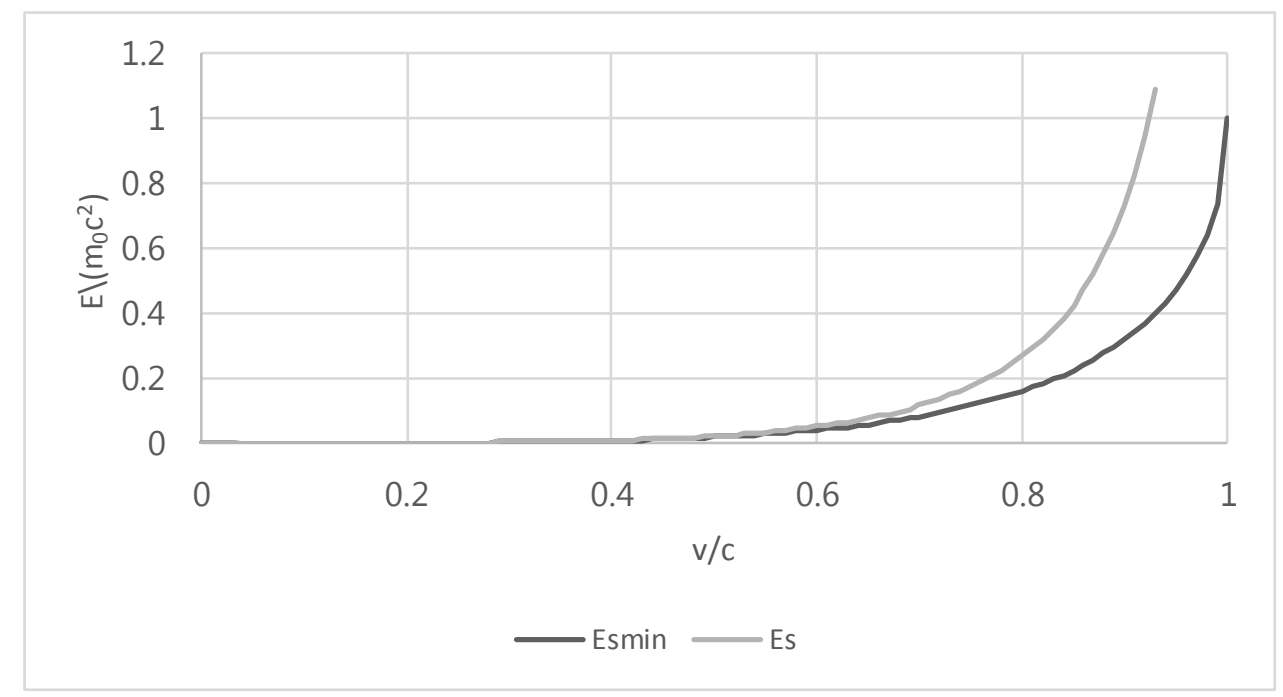

Fig. 11 Graph presenting the dependence of $\frac{E_{S_{\min }}\left(\frac{v}{c}\right)}{m_{0} c^{2}}$ and $\frac{E_{S}\left(\frac{v}{c}\right)}{m_{0} c^{2}}$

\footnotetext{
${ }^{7}$ For such case, $E_{\Delta J}$ is close to zero.

${ }^{8}$ A spacecraft with stabilisation of the orientation of spatial channels does not deform the boundary hypersurfaces in large areas of the space and therefore, the deformation energy of boundary hypersurfaces is finite.
} 


\section{Division of rocket engines}

Direct cause of motion is asymmetrical deformation of the boundary hypersurfaces ${ }^{\alpha} \mathcal{N}^{\alpha}$ and ${ }^{\beta} \boldsymbol{N}^{\beta}$ by a macroscopic object, which directly results from the theory entitled "Theory of Space" (Sobolewski D. S., Theory of Space, 2016) (Sobolewski D. S., Theory of Space, 2017).

Thus, the results obtained in the previous chapter, and particularly the eight orders lower energy $E_{S}$ needed to deform the boundary hypersurfaces ${ }^{\alpha} \aleph^{\alpha}$ and ${ }^{\beta} \aleph^{\beta}$ than the energy required to change the orientation of the spatial channels, from which the macroscopic object is built, are astounding.

This means that after mastering the technology of asymmetric deformation of boundary hypersurfaces, with simultaneous maintaining of the orientation of spatial channels, from which the spacecraft is built, it would be possible to reach distant planets, not only at the expense of significantly lower energy, but even in a significantly shorter time, due to the possibility of achieving velocities of thousands of kilometres per second.

For example, the acceleration of a spacecraft with the mass $\mathrm{m}_{0}=10^{3} \mathrm{~kg}$ to the velocity $v=10^{6} \frac{\mathrm{m}}{\mathrm{s}}$ would only require the consumption of energy needed for deforming the boundary hypersurfaces $\mathrm{E}_{\mathrm{S}_{\min }}$, which we will calculate from the formula (26):

$$
\begin{array}{r}
E_{S_{\text {min }}}=\mathrm{m}_{0} \mathrm{c}^{2}\left(1-\cos \Theta_{\mathrm{v}}\right)^{2}=9 * 10^{19}\left(1-\sqrt{1-\left(\frac{10^{6}}{3 * 10^{8}}\right)^{2}}\right)^{2} \\
=9 * 10^{19}(1-0.9999944444)^{2} \approx 2.78 * 10^{9} \mathrm{~J}
\end{array}
$$

i.e. approximately $2.78 \mathrm{GJ}$, which is the energy two orders lower than the energy needed to change the orientation of space channels of the same spacecraft when accelerating to the velocity $30 \frac{\mathrm{km}}{\mathrm{s}}$.

In the light of the above, let us check how much time it would take to travel with such spacecraft from Earth to Mars, which are separated from each other by a distance from $54 * 10^{9} \mathrm{~m}$ to $103 * 10^{9} \mathrm{~m}$ :

$$
\begin{aligned}
& \mathrm{t}_{\text {min }}=\frac{s}{v} \approx \frac{54 * 10^{9} \mathrm{~m}}{10^{6} \frac{\mathrm{m}}{\mathrm{s}}} \approx 54 * 10^{3} \mathrm{~s}=15 \mathrm{~h} \\
& \mathrm{t}_{\text {max }}=\frac{s}{v} \approx \frac{103 * 10^{9} \mathrm{~m}}{10^{6} \frac{\mathrm{m}}{\mathrm{s}}} \approx 103 * 10^{3} \mathrm{~s}=28 \mathrm{~h} 36^{\prime} 40 \mathrm{~s}
\end{aligned}
$$

In connection with the above-mentioned considerations, we will introduce division of rocket engines as follows:

- Jet engines,

- HTS engines,

- HTS engines with stabilisation.

Jet rocket engines change the orientation of space channels, from which the spacecraft is constructed, and as a result of that, the deformation of boundary hypersurfaces ${ }^{\beta} \mathrm{N}^{\beta}$ and ${ }^{\alpha} \mathrm{N}^{\alpha}$ changes. On the other hand, rocket HTS engines change the deformation of boundary hypersurfaces ${ }^{\beta} \mathrm{N}^{\beta}$ and ${ }^{\alpha} \aleph^{\alpha}$ of the spacecraft, which results in a change in the orientation of its spatial channels. Rocket HTS engines with stabilisation are HTS engines, which by changing the deformation of boundary hypersurfaces ${ }^{\beta} \mathrm{N}^{\beta}$ and ${ }^{\alpha} \boldsymbol{\aleph}^{\alpha}$, do not change the orientation of spatial channels from which the spacecraft is constructed. 
In short, the jet rocket engines change energy $E_{\Delta J}$, while the HTS engines change energy $E_{S}$ and HTS engines with stabilisation do not change energy $E_{\Delta J}$ of the spacecraft.

\section{Interaction of photons ${ }^{\beta} \gamma$ with the boundary hypersurface ${ }^{\beta}{ }^{\beta}$}

As it was mentioned in the previous chapter, the HTS Rocket Engines do not have to eject elementary particles, in order to obtain thrust force, like in the case of jet engines, which will be demonstrated during the discussion of the principle of operation of the "HTS Photonic Rocket Engine", patented by the co-authors of this article (Poland Patent nr P.421517, 2017).

However, prior to the discussion of the principle of operation of the patented engine (Poland Patent $\mathrm{nr}$ P.421517, 2017), we have to remind the properties of alpha ${ }^{\alpha} \gamma$ and beta ${ }^{\beta} \gamma$ photons, as well as their interaction with the deformed boundary hypersurfaces ${ }^{\beta} \aleph^{\beta}$ and ${ }^{\alpha} \aleph^{\alpha}$, which were presented in the publication entitled "Geometry of the Dark Matter and Preliminary Analysis of Alpha and Beta Photons' Properties Based on Theory of Space" (Sobolewski, Sobolewski i Sobolewski, 2017).

It turns out that beta ${ }^{\beta} \gamma$ photons, identified with the photons known to us, which are essentially vortical space disturbances that connect boundary hypersurfaces ${ }^{\beta} \aleph^{\beta}$, create four-dimensional spatial channels (tubes), and this is illustrated in Fig. 1 - they are marked as ${ }^{\beta} \gamma_{1},{ }^{\beta} \gamma_{2}$.

As a result of self-interaction with itself, the photon ${ }^{\beta} \gamma$ rotates, and its ends oscillate around the equilibrium positions, and this is manifested by a periodic increase in the advantage of the electric or magnetic field (Sobolewski, Sobolewski i Sobolewski, 2017).

Interestingly, such vortical disturbances can be seen even in a pool of water (Cowern, 2019), which is presented in the Fig. 12:

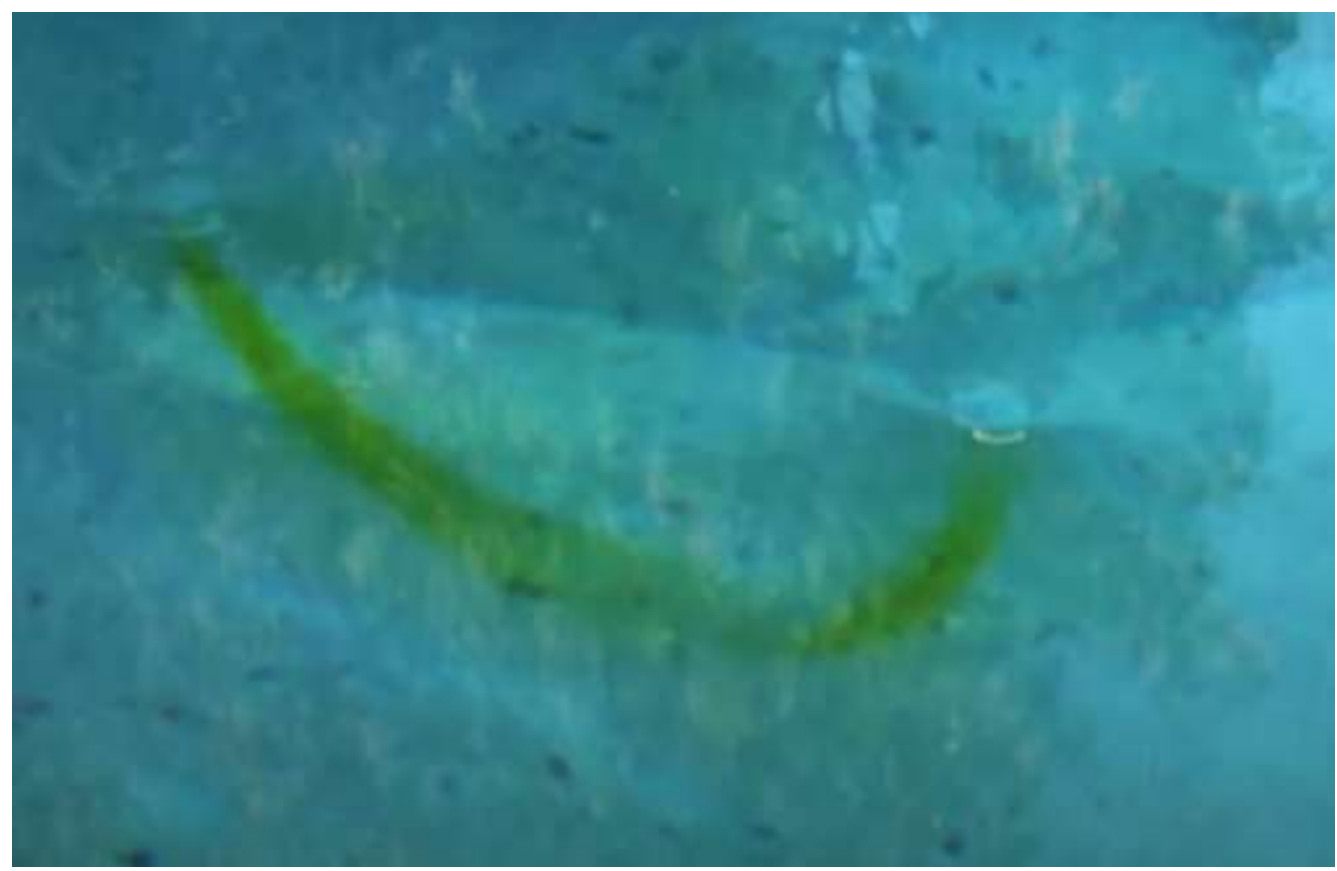

Fig. 12 Vortex in a pool of water

In the case of a vortex in water, in contrast to the real four-dimensional space, in which four-dimensional boundary layers have three-dimensional boundary hypersurfaces that are elastic, we deal with three dimensional 
space and two-dimensional boundary hypersurface, the elasticity of which is close to zero due to the very low surface tension of water 9 .

Now, let us analyse the interaction of beta ${ }^{\beta} \gamma$ photons with the boundary hypersurface ${ }^{\beta}{ }^{\beta}$ deformed by a moving macroscopic rigid body. For this purpose, we will set a laser, which is a source of photons with the initial frequency $v_{0}$, in such manner that will make them propagate in the direction of the positive axis $e_{1}$ behind the macroscopic object with the mass $m_{0}$, which also moves in the direction of the positive axis $e_{1}$ at velocity $v$.

The described experiment can be presented in a simplified manner by analysing the projection on the plane $\mathrm{L}\left(e_{1}, e_{4}\right)$ - which is presented the Fig. 13.

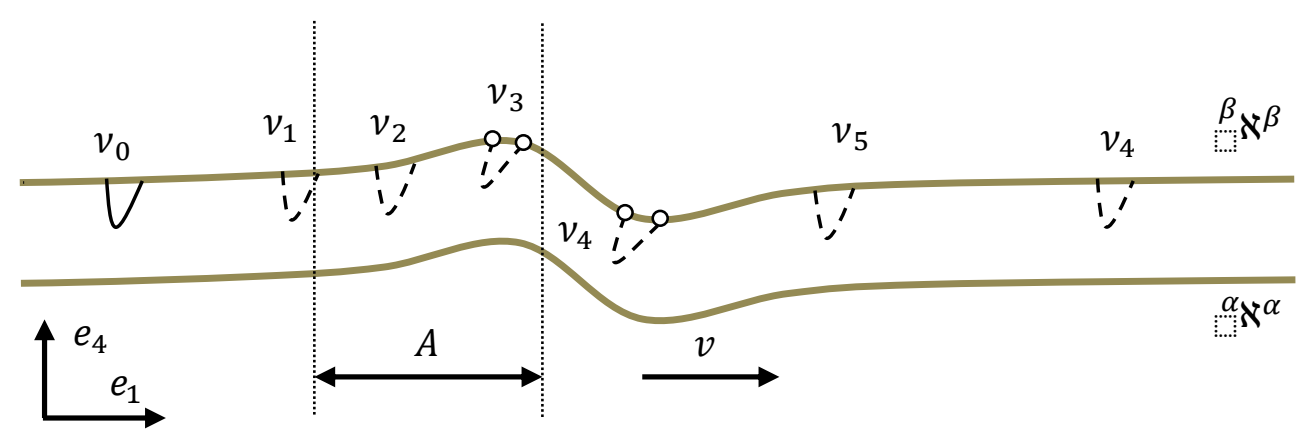

Fig. 13 Photons $v_{0}$ that propagate in the positive direction of the axis $e_{1}$, passing through the deformed boundary hypersurfaces of the macroscopic system with mass $m_{0}$, which moves in the direction of the positive axis $e_{1}$ at velocity $v$ - overview figure.

When reaching the area of deformed boundary hypersurfaces ${ }^{\beta} \aleph^{\beta}$, through the macroscopic body, photons with the frequency $v_{0}$ interact with them by changing their frequency (Sobolewski, Sobolewski i Sobolewski, 2017). As a result of this interaction, deformation of the boundary hypersurface ${ }^{\beta} \mathrm{N}^{\beta}$ also changes, which results directly from the conservation of energy principle:

$$
\Delta \mathrm{E}_{\mathrm{S} \beta}=\mathrm{h} \Delta v
$$

where $E_{S \beta}$ is the deformation energy of boundary hypersurface ${ }^{\beta}{ }^{\beta}$. However, we will assume that $h \Delta v=\Delta E_{S}$, because deformation of the boundary hypersurface ${ }^{\beta} \aleph^{\beta}$ also deforms the boundary hypersurface ${ }^{\alpha} \aleph^{\alpha}$ :

$$
\Delta \mathrm{E}_{\mathrm{S}}=\mathrm{h} \Delta v
$$

Considerations in the article entitled "Geometry of the Dark Matter and Preliminary Analysis of Alpha and Beta Photons' Properties Based on Theory of Space" (Sobolewski, Sobolewski i Sobolewski, 2017) indicate that beta photons ${ }^{\beta} \gamma$, while passing through areas of the space with deformed boundary hypersurfaces ${ }^{\beta} \aleph^{\beta}$, increase their energy in convex areas of the deformation (curvature $\kappa(s)$ is less than zero $\kappa(s)<0$ ) and decrease it in concave areas of the deformation (curvature $\kappa(s)$ is greater than zero $\kappa(s)>0$ ).

\footnotetext{
${ }^{9}$ We assume that the spacecraft is a rigid body.
} 


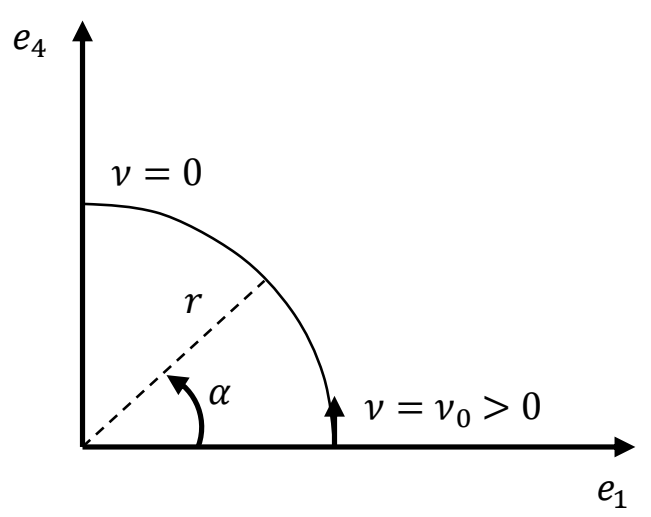

Fig. 14 While passing through curved area of the space, photon with frequency $v_{0}$ completely loses its energy, regardless of the radius of the curvature $r$.

Regardless of the deformation curvature of boundary hypersurface ${ }^{\beta} \mathrm{N}^{\beta}$, we want the frequency of the beta photon ${ }^{\beta} \gamma$ to be equal to zero after passing through the area of $\frac{1}{4}$ of the sphere circumference, along the geodesics e.g. of the meridian, which directly results from the theory entitled "Theory of Space" (Sobolewski D. S., Theory of Space, 2016) (Sobolewski D. S., Theory of Space, 2017).

Thus, the following formula for determining the change in photon frequency is introduced ${ }^{10}$ :

$$
\frac{v}{v_{0}} \equiv 1-\frac{2}{\pi} \int_{s_{0}}^{s_{1}} \kappa(s) d s
$$

where the curvature $\kappa$ :

$$
\kappa \equiv \lim _{\Delta S \rightarrow 0} \frac{\Delta \alpha}{\Delta S}
$$

which for the parametrically described function is expressed by the following formula:

$$
\kappa(t)=\frac{y^{\prime \prime}(t) x^{\prime}(t)-x^{\prime \prime}(t) y^{\prime}(t)}{\left(x^{\prime}(t)^{2}+y^{\prime}(t)^{2}\right)^{\frac{3}{2}}}
$$

Let us verify, whether the formula for frequency change $\frac{v}{v_{0}}$, which was defined by us, despite its triviality in the scope of the curve being a section of a circle, leads to the expected results.

For this purpose, let us apply the following parameterisation of the curve:

$$
\begin{aligned}
& \mathrm{x}_{1}\left(\frac{s}{r}\right)=\mathrm{r} * \cos \left(\frac{s}{r}\right) \\
& \mathrm{x}_{4}\left(\frac{s}{r}\right)=\mathrm{r} * \sin \left(\frac{S}{r}\right)
\end{aligned}
$$

Derivatives $\mathrm{x}_{1}(\alpha)$ and $\mathrm{x}_{4}(\alpha)$ :

\footnotetext{
${ }^{10}$ In subsequent publications, we will introduce more general formulas and the geometric concept of four-dimensional curvature of the boundary hypersurface.
} 


$$
\begin{gathered}
\mathrm{x}_{1}{ }^{\prime}\left(\frac{S}{r}\right)=-\sin \left(\frac{S}{r}\right) \\
\mathrm{x}_{4}{ }^{\prime}\left(\frac{S}{r}\right)=\cos \left(\frac{S}{r}\right) \\
\mathrm{x}_{1}{ }^{\prime \prime}\left(\frac{S}{r}\right)=-\frac{1}{r} * \cos \left(\frac{S}{r}\right) \\
\mathrm{x}_{4}{ }^{\prime \prime}\left(\frac{S}{r}\right)=-\frac{1}{r} * \sin \left(\frac{S}{r}\right)
\end{gathered}
$$

Therefore, the curvature $\kappa$ is equal to:

$$
\kappa=\frac{\frac{1}{r} * \sin ^{2}\left(\frac{S}{r}\right)+\frac{1}{r} * \cos ^{2}\left(\frac{S}{r}\right)}{\left(\sin ^{2}\left(\frac{S}{r}\right)+\cos ^{2}\left(\frac{S}{r}\right)\right)^{\frac{3}{2}}}=\frac{1}{r}
$$

After substitution to the formula (31), the result is as follows:

$$
\frac{v}{v_{0}}=1-\frac{2}{\pi} \int_{0}^{\frac{\pi}{2} r} \kappa(s) d s=1-\frac{2}{\pi} *\left(\frac{1}{r} * \frac{\pi}{2} r\right)=1-1=0
$$

Ultimately, we obtain the final frequency as equal to zero:

$$
v=0
$$

which means that the area of the Universe, which is visible to us, is limited to half a sphere with the radius of $W_{\Re}=4040.33 \mathrm{Mpc}$. (Sobolewski, Sobolewski i Sobolewski, 2017) (Sobolewski D. S., Theory of Space, 2016) (Sobolewski D. S., Theory of Space, 2017).

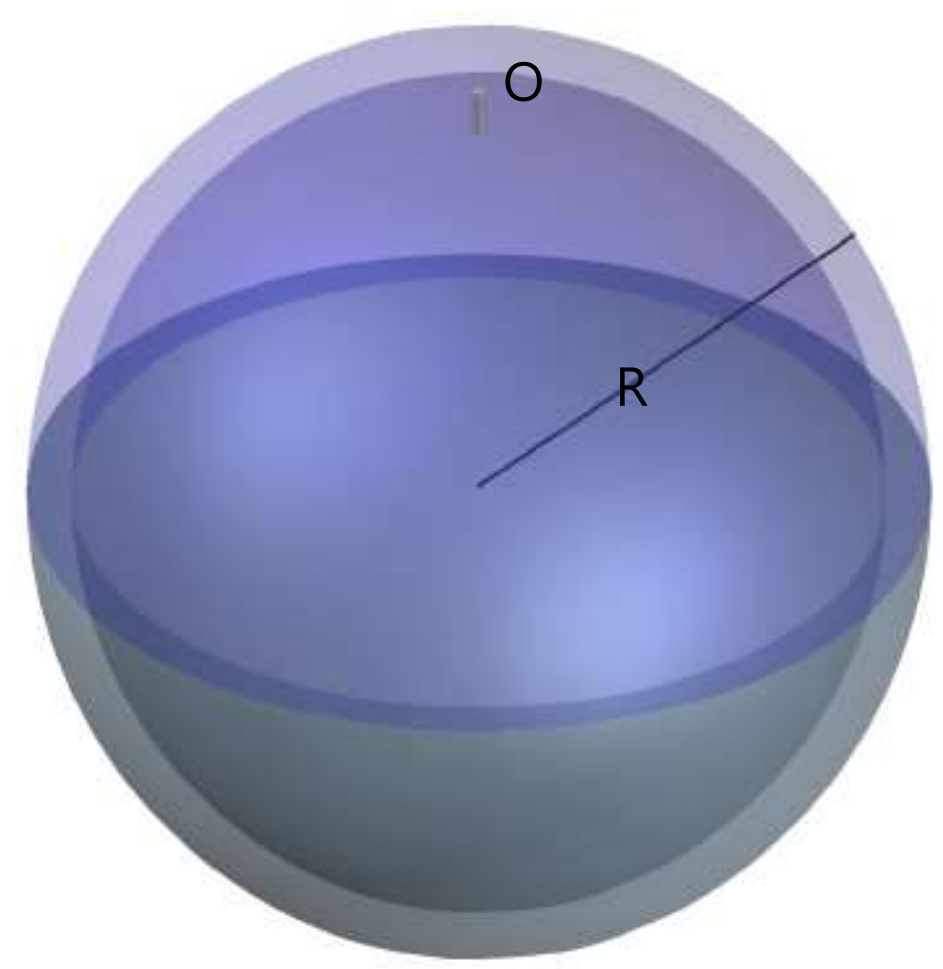

Fig. 15 Visible area of the Universe from the 0 point. 
By applying the Doppler law in the relativistic form, the result is as follows:

$$
\begin{aligned}
& \frac{\Delta \lambda}{\lambda} \approx \frac{v}{c} \\
& \frac{\Delta \lambda * c}{\lambda * c}=\frac{\left(\lambda-\lambda_{0}\right)}{c} v_{0}=\left(\frac{1}{v}-\frac{1}{v_{0}}\right) v_{0}=\frac{v_{0}}{v}-1 \approx \frac{v}{c} \\
& v_{0}-v \approx v \frac{v}{c} \\
& v_{0} \approx v+v \frac{v}{c}=v\left(1+\frac{v}{c}\right) \\
& v=\frac{v_{0}}{\left(1+\frac{v}{c}\right)}
\end{aligned}
$$

By expanding the equation (39) into the Maclaurin series in relation to $\frac{v}{c}$, the result is as follows:

$$
\begin{aligned}
& v \approx v_{0}\left(1-\frac{v}{c}+O\left(\frac{v^{2}}{c^{2}}\right)\right) \\
& \frac{v}{v_{0}} \approx 1-\frac{v}{c}
\end{aligned}
$$

By substituting right side of the equation (40) to the equation (31), the result is as follows:

$$
\begin{gathered}
1-\frac{v}{c}=1-\frac{2}{\pi} \int_{s_{0}}^{s_{1}} \kappa(s) d s \\
\frac{v}{c}=\frac{2}{\pi} \int_{s_{0}}^{s_{1}} \kappa(s) d s \\
\sin \Theta_{v}=\frac{2}{\pi} \int_{s_{0}}^{s_{1}} \kappa(s) d s
\end{gathered}
$$

Equation (43) means that the deformation of boundary hypersurfaces behind the moving object at velocity $v$, which is demonstrated in Fig. 13, in addition to the concave area, must have a convex area with the curvature $\kappa(s)<0$, due to the following inequality:

$$
\sin \alpha<\alpha
$$

\section{HTS Photonic Rocket Engine with the use of photons ${ }^{\beta} \gamma$}

The principle of operation of the HTS Photonic Rocket Engines consists of using the phenomenon of photon interaction with boundary hypersurfaces ${ }^{\beta} \aleph^{\beta}$ and ${ }^{\alpha} \aleph^{\alpha}$, which by transferring energy to them, in accordance with formula (30), increase their deformation. In turn, increase in the deformation energy of boundary hypersurfaces increases the spacecraft's velocity in accordance with the formula (14).

The first attempt of constructing this type of engines was undertaken by the co-authors of this article in the patent application entitled "HTS Photonic Rocket Engine", registered in the Patent Office of the Republic of Poland under the number P.421517, the diagram of which is presented in the Fig. 16. 


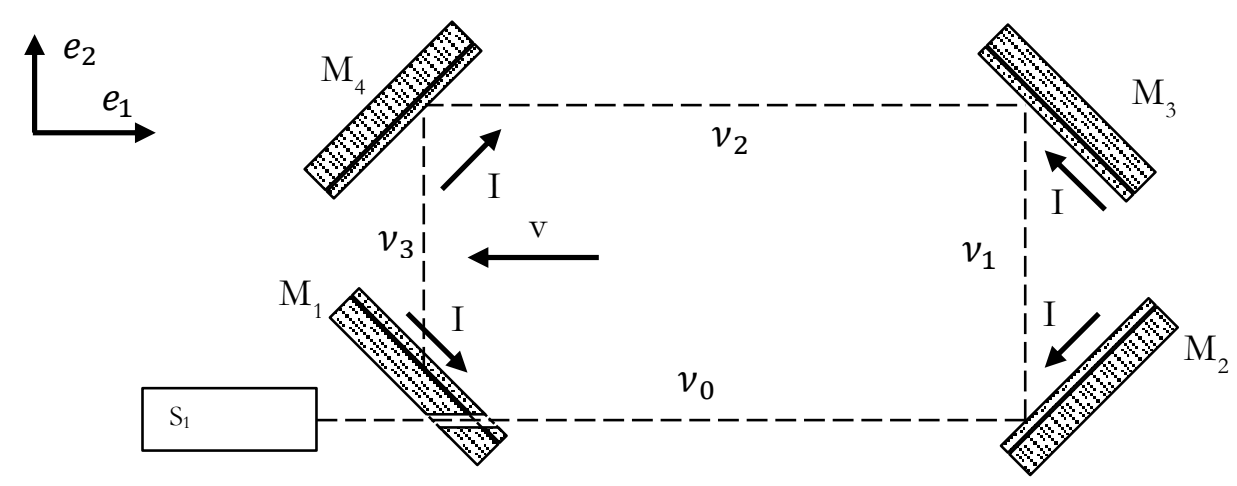

Fig. 16 Diagram of the first version of the rocket HTS Photonic Rocket Engine.

A spacecraft with mounted HTS engine moves at the velocity $v$ opposite to the beam of photon flux, with frequency $v_{0}$ generated by the laser $S_{1}$, which is marked in the Fig. 16 .

Mirrors $M_{1}, M_{2}, M_{3}, M_{4}$ form the optical system of the rocket HTS engine, circulated many times by photons emitted by the laser $S_{1}$ without interference, because the laser beam's path changes with each cycle. Mirrors have graphene ${ }^{11}$ layers isolated from each other (e.g. with the use of thin layers of glass), through which the electric current $I$ flows.

Electrons moving in graphene layers (Novoselov $\mathrm{i}$ inni, 2005) at velocities reaching up to $\frac{1}{300} c$ deform the boundary hypersurfaces, and due to their component $e_{1}$, they have the direction opposite to the vector of rocket velocity $v$ on the mirrors $M_{2}, M_{3}, 12$, while on the mirrors $M_{1}, M_{4}$ they have the direction compliant with the vector of rocket velocity $v$. This means that photons reflected from the mirror $M_{2}$, covered with graphene layers, pass through the area of deformed boundary hypersurfaces $A$ marked in Fig. 13, in which they reduce their frequency $v_{1}<v_{0}$, as mentioned in the previous chapters ${ }^{13}$.

Reduction of the frequency of photons in the graphene layers prior to transferring the momentum to the mirror $M_{2}$ allows for reduction of their interaction with the mirror despite reflection, therefore the photon beam can be used after reflection from the mirror $M_{3}$ for directing to the mirror $M_{4}$ that propels the rocket. Prior to reflection from the mirror $M_{4}$, the photons also pass through the deformed boundary hypersurfaces $A$, which are marked in the Fig. 13.

Therefore, while passing through the deformed boundary hypersurfaces (as a result of electrons moving at velocities similar to the velocity of light) in graphene layers on the mirrors $M_{1}, M_{4}$, the photons will reduce their energy, at the same time increasing the deformation of boundary hypersurfaces, which, as we expect, will increase the deformation of spacecraft's boundary hypersurfaces that will increase the spacecraft's velocity, in accordance with the formula (14).

While using laser with power $P_{l}$, as a result of a single reflection of the laser beam from the mirror $M_{4}$, we can obtain thrust force $\mathrm{F}_{T}$ equal to:

\footnotetext{
${ }^{11}$ We use graphene due to the velocities achieved by the conduction electrons equal to $\frac{1}{300} c$ and the possibility to create a layer of such electrons with a large surface, with low energy consumption (Novoselov i inni, 2005).

12 Electrons moving in the graphene layers can be also compared to the receding mirrors, due to their velocity component $e_{1}$.
} 


$$
\mathrm{F}_{T}=\mathrm{n} \frac{\Delta p}{\Delta \mathrm{t}}=\frac{P_{l} \Delta \mathrm{t}}{h v} \frac{h v}{\Delta \mathrm{tc}}=\frac{P_{l}}{\mathrm{c}}
$$

For example, by using laser with power $P_{l}=1000 \mathrm{~W}$, we obtain thrust force $\mathrm{F}_{T} \cong 3,34 \mu \mathrm{N}$ :

$$
\mathrm{F}_{T}=\frac{1000 \mathrm{~W}}{299792458 \frac{\mathrm{m}}{\mathrm{s}}}=3,34 * 10^{-7} \mathrm{~N} \cong 3,34 \mu \mathrm{N}
$$

Thus, by reflecting the light beam in the system presented in schematic Fig. 10 even up to 1000 times, we will achieve a thrust force equal to $\mathrm{F}_{T} \cong 3,34 \mathrm{mN}$. In order to increase the thrust force, graphene layers were used on the mirrors $M_{1}, M_{2}, M_{3}, M_{4}$, through which the electric current $I$ flows, as marked in the figure.

We expect that the additional deformation of boundary hypersurfaces in the area of graphene layers will strengthen the deformation of rocket's boundary hypersurfaces, while increasing its velocity and thrust force of the rocket HTS engine.

The application of HTS engine, along with stabilisation of the orientation of spatial channels, from which the spacecraft is built, makes it possible to significantly reduce the energy required for its acceleration, and therefore the rocket HTS engine may have a thrust several orders of magnitude lower than traditional jet engines, which results from the formula (40).

It should be emphasized that the above-mentioned structure of the rocket HTS engine, despite being a step in the right direction due to the presented theoretical basis, should be perceived as the first attempt to build this type of engines, which requires further research works.

In the patent application " HTS Photonic Rocket Engine", registered under the number P.421517, the mirrors presented in the below Fig. 17 were used:
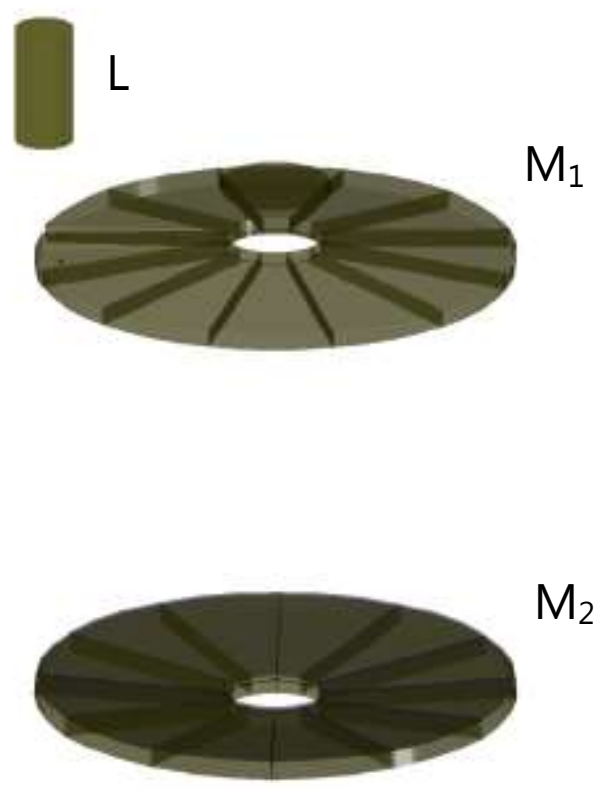

Fig. 17 Optical system of the patent application "HTS Photonic Rocket Engine" registered under the number P.421517 (Poland Patent nr P.421517, 2017). 


\section{Rocket HTS engines with stabilisation}

Theoretical considerations presented above demonstrated that the deformation energy of boundary hypersurfaces of the moving objects is significantly lower, than the energy of changing the orientation of spatial channels, which directly results from the formulas (13), (26) and (15):

$$
\frac{E_{\Delta J}}{E_{S_{\text {min }}}}=\frac{\mathrm{m}_{0} \mathrm{c}^{2}\left(1-\cos \Theta_{\mathrm{v}}\right)}{\mathrm{m}_{0} \mathrm{c}^{2}\left(\cos \Theta_{\mathrm{v}}-1\right)^{2}}=\frac{1}{1-\cos \Theta_{\mathrm{v}}}=\frac{1}{1-\sqrt{1-\frac{v^{2}}{c^{2}}}}
$$

The graph of dependency $\frac{E_{\Delta \mathrm{J}}}{E_{S_{\min }}}(v)$ is presented below:

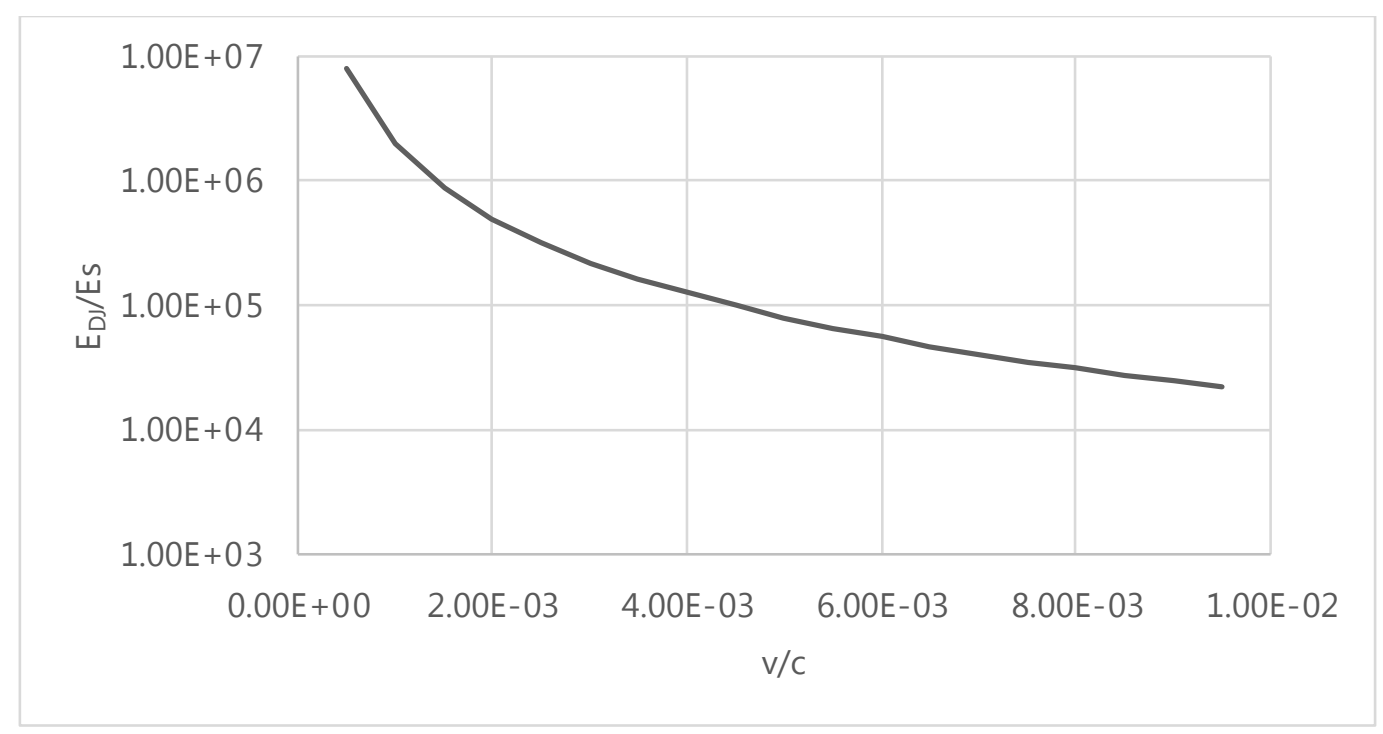

Fig. 18 Graph of dependence $\frac{E_{\Delta J}}{E_{S_{\min }}}\left(\frac{v}{c}\right)$

At this point, the following question arises: is it possible to "stabilise" the spacecraft using this method and in the manner that will not change the angle of inclination of the spatial channels, from which it is constructed, while reducing the energy necessary to accelerate it, even up to a million times?

Currently, we do not know the answer to that question, however development of the technology that stabilises the spatial channels of the rocket would allow humanity to reach distant planets of our galaxy, even those located light years away from Earth, and this results not only from possibility of a spacecraft to achieve relativistic velocities, but also from reduction of overloads inside the rocket during its acceleration, and even during rapid reduction of velocity.

It is necessary to note quickly growing quotient of energy $\frac{\mathrm{E}_{\Delta \mathrm{J}}}{E_{S_{\min }}}$ for low velocities, which approaches infinity. Therefore, mastering of the technology of constructing HTS engines with stabilisation would not only allow for reduction of energy required to accelerate cars, airplanes and trains, but above all, it would improve traffic safety due to manoeuvre capabilities, i.e. the ability to quickly change their acceleration without the consequences of overloads observed inside the vehicle.

Unfortunately, due to the motion of these vehicles in the Earth's atmosphere, as well as in the variable gravitational field, and after taking into account the friction and undoubtedly a very high price for HTS engines with stabilisation, we come to the conclusion that the possible application of such technology in terrestrial conditions would be probably limited only to ensuring safety to the passengers. 
Currently, we consider the theoretical possibilities of building HTS engines with stabilisation. It is certain that in order to stabilise the orientation of spatial channels of the rocket, additional energy would be required for "stabilisation" of the orientation of spatial channels of the rocket, which we will mark with $E_{S t}$.

In the worst case, stabilisation of the orientation of spatial channels would be necessary for the time of rocket motion in time $t$. Moreover, energy $E_{S t}$ required for stabilisation of the orientation of spatial channels cannot be lower than the deformation energy of boundary hypersurfaces $E_{S_{\min ^{\prime}}}$ and therefore, depending on the applied technology, energy $E_{S t}$ satisfies the following inequalities:

$$
E_{S_{\text {min }}} \leq E_{S t} \leq E_{S_{\text {min }}} * t
$$

Thus, in the worst case, the energy required for the stabilisation of spatial channels $E_{S t}$ depends on the time of rocket flight, and in the best case, it is approximate to energy $E_{S_{\min }}$.

The total energy $E_{T}$ that is necessary to accelerate the spacecraft to velocity $v$ satisfies the following inequalities:

$$
\begin{aligned}
& E_{S_{\min }}+E_{S t} \leq E_{T} \leq E_{S_{\min }}+E_{S_{\min }} * t \\
& 2 E_{S_{\min }} \leq E_{T} \leq(1+t) E_{S_{\min }}
\end{aligned}
$$

The first approximation of the rocket structure with stabilisation of the orientation of spatial channels would be the application of graphene layers as a spacecraft shell, through which the current would flow in the manner marked in the below Fig. 19. However, the graphene layers should be electrically isolated from each other ${ }^{14}$.

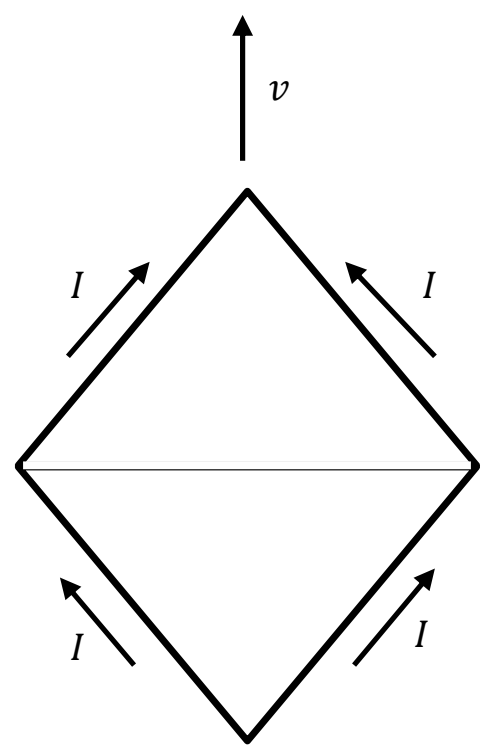

Fig. 19 Overview figure of a spacecraft covered with the layers of graphene, through which the electric current $I$ flows.

\footnotetext{
${ }^{14}$ In the subsequent publications, we will return to the subject of estimating the number of graphene lay ers used to construct the rocket shield, which will also allow to determine the current flowing through the rocket shell and energy $E_{S t}$.
} 
For the case presented by us in the Fig. 19, we expect the deformation of boundary hypersurfaces presented in the Fig. 20.

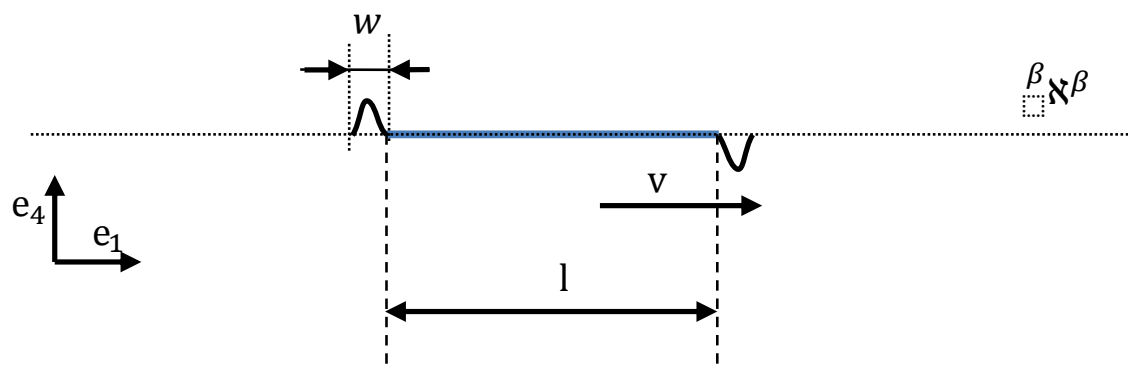

Fig. 20 Spacecraft with the mass $m_{0}$ that moves at the velocity $v$, covered with the layers of graphene, through which the electric current $I$ flows - overview figure

Unfortunately, we do not know, among others, the value of deformation of the boundary hypersurfaces $w$, which can range from one millimetre to hundreds of metres, as well as the correctness of assumptions, thus our considerations should be perceived as the first approximation of this issue, on the way to the construction of not only new generation rocket engines, but also spacecraft that can travel at the velocities similar to the velocity of light.

\section{Conclusions}

This article presents theoretical basics of new technologies aimed at constructing rocket engines and spacecraft, along with the first examples of their implementation, which reveal that interplanetary travel is possible even to the planets located light years away from us, due to the possibility of achieving relativistic velocities by a spacecraft.

It turns out that due to stabilisation of the orientation of spatial channels, from which the spacecraft is constructed, it is possible not only to reduce the value of energy required to accelerate a spacecraft to relativistic velocities by several orders of magnitude, but even avoid the effects of overload.

Moreover, the works on the technologies of stabilisation of the spacecraft's spatial channels should also contribute to reducing the risk of travel associated with overloads that, as it turns out, can be eliminated.

In order to illustrate the possibilities of the new technology, let us imagine a passenger car equipped with the technology of stabilising spatial channels, which travels at a velocity of several hundred kilometres per hour, and suddenly hits a concrete wall and stops, however it does not lead to overloads inside the car or even to its damage.

Knowledge shared in this article on matter, gravitational and fluctuation waves in conjunction with knowledge embedded in "Theory of Space" (Sobolewski D. S., Theory of Space, 2016) (Sobolewski D. S., Theory of Space, 2017) and in article "Geometry of the Dark Matter and Preliminary Analysis of Alpha and Beta Photons" (Sobolewski, Sobolewski i Sobolewski, 2017) is also grounds for the creation of four-dimensional quantum mechanics, describing all of the types of interactions.

Due to the technology of stabilisation of the orientation of spatial channels, it may even turn out that it is possible to use the gravitational potential energy of a spacecraft to propel it, which directly results from the above considerations, as well as the essence of gravitational interactions revealed in the theory entitled "Theory of Space". (Sobolewski D. S., Theory of Space, 2016) (Sobolewski D. S., Theory of Space, 2017). 
Rocket HTS engines are marked with the HTS's company logo, which is presented in the Fig. 21.

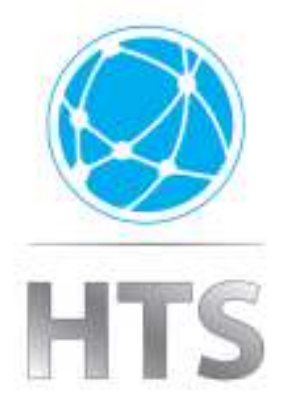

Fig. 21 Company logo of HTS High Technology Solutions, as well as rocket HTS engines and HTS engines with stabilisation.

\section{References}

1. Cowern, D. (2019, 5 29). Physics Girl in a YouTube channel. Retrieved from https://www.youtube.com/watch?v=_18avidXxqY

2. Novoselov, K., Geim, A., Morozov, S., Jiang, D., Katsnelson, M., Grigorieva, I., . . Firsov, A. (2005). Two dimensional gas of massless Dirac fermions in graphene. Nature, 438, 197-200.

3. Sobolewska, N. J., Sobolewska, J. P., Sobolewski, M. J., Sobolewski, M. A., \& Sobolewski, D. S. (2017, maj 7). Poland Patent No. P.421517.

4. Sobolewski, D. S. (2016). Theory of Space. Cambridge International Science Publishing Ltd. and Viva Books Private Limited.

5. Sobolewski, D. S. (2017). Theory of Space. HTS High Technology Solutions.

6. Sobolewski, M. J., Sobolewski, M. A., \& Sobolewski, D. S. (2017, July). Geometry of the Dark Matter and Preliminary Analysis of Alpha and Beta Photons' Properties Based on Theory of Space. International Journal of Innovative Research in Science. 